\title{
DÜBLIN
}

Technological University Dublin

ARROW@TU Dublin

2008-01-01

\section{RFID: an Ideal Technology for Ubiquitous Computing?}

\author{
Ciaran O'Driscoll \\ Technological University Dublin, ciaran.odriscoll@tudublin.ie \\ Daniel MacCormac \\ Technological University Dublin, dan.maccormac@comp.dit.ie \\ Mark Deegan \\ Technological University Dublin, mark.deegan@tudublin.ie
}

See next page for additional authors

Follow this and additional works at: https://arrow.tudublin.ie/engschececon

Part of the Computer Engineering Commons

\section{Recommended Citation}

O'Driscoll, C., MacCormac, D., Deegan, M., Mtenzi, F., O'Shea, B.: RFID: An Ideal Technology for Ubiquitous Computing? 5th International Conference on Ubiquitous Intelligence and Computing 2008, UIC '08, Oslo University College, Norway, 2008.

This Conference Paper is brought to you for free and open access by the School of Electrical and Electronic Engineering at ARROW@TU Dublin. It has been accepted for inclusion in Conference papers by an authorized administrator of ARROW@TU Dublin. For more information, please contact arrow.admin@tudublin.ie, aisling.coyne@tudublin.ie,gerard.connolly@tudublin.ie.

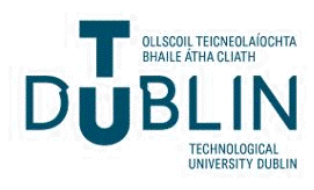




\section{Authors}

Ciaran O'Driscoll, Daniel MacCormac, Mark Deegan, Fredrick Mtenzi, and Brendan O'Shea 
$1-1-2008$

\section{RFID: An Ideal Technology for Ubiquitous Computing?}

Ciaran O'Driscoll

Dublin Institute of Technology, ciaran.odriscoll@dit.ie

Daniel MacCormac Mr.

Dublin Institute of Technology, dan.maccormac@comp.dit.ie

Mark Deegan Mr.

Dublin Institute of Technology, mark.deegan@dit.ie

Fred Mtenzi Dr.

Dublin Institute of Technology, fred.mtenzi@dit.ie

Brendan O'Shea Prof.

Dublin Institute of Technology, brendan.oshea@dit.ie 


\title{
RFID: An Ideal Technology for Ubiquitous Computing?
}

\author{
Ciaran O’Driscoll, Daniel MacCormac, Mark Deegan, Fred Mtenzi, Brendan \\ O'Shea \\ Dublin Institute of Technology, Kevin Street, Dublin 8, Ireland \\ ciaran.odriscoll@dit.ie \\ \{dan.maccormac, mark.deegan, fred.mtenzi, brendan.oshea\}@comp.dit.ie
}

\begin{abstract}
This paper presents a review of RFID based approaches used for the development of smart spaces and smart objects. We explore approaches that enable RFID technology to make the transition from the recognized applications such as retail to ubiquitous computing, in which computers and technology fade into the background of day to day life. In this paper we present the case for the use of RFID technology as a key technology of ubiquitous computing due to its ability to embed itself in everday objects and spaces. Frameworks to support the operation of RFID-based smart objects and spaces are discussed and key design concepts identified. Conceptual frameworks, based on academic research, and deployed frameworks based on real world implementations are reviewed and the potential for RFID as a truly ubiquitous technology is considered and presented.
\end{abstract}

\section{INTRODUCTION}

The case for RFID in retail [1] and logistics [2] has been clearly identified and is being actively pursued by many companies. RFID technology, in association with Electronic Product Codes (EPC) [3], has been identified as an enabler in Supply Chain Management (SCM) in the retail case study presented in [1]. There is a clear financial motivation for companies like Wal-mart to have suppliers provide RFID tagging on products. In 2003, Roberti in [4] refers to estimates by Sanford C. Bernstein \& Co., a New York investment research house, that Wal-Mart could save nearly 8.4 billion USD per year when RFID is fully deployed throughout its supply chain and in stores.

The potential for RFID beyond the scope of Retail and SCM into the area of ubiquitous computing is being widely researched and this is an area of growing interest.

Ubiquitous computing involves computers and technology that blend seamlessly into day to day living. Weiser [5] introduced the concept of ubiquitous computing in 1991, he begins his article with:

"The most profound technologies are those that disappear. They weave themselves into the fabric of everyday life until they are indistinguishable from it." 
This description of a disappearing technology can clearly be applied to the trend in RFID technology development. The technology is maturing, size and form factors are reducing, and the cost of passive tags is now in the order of 10s of cents. As RFID is becoming such a ubiquitous technology, it is of particular interest to review its use in the area of Smart Spaces $[4,6]$ for ubiquitous computing.

\section{MOTIVATION}

The motivation for this paper and our current research is to identify and determine technologies that are suitable to support the development of ubiquitous computing environments. This paper focuses on the potential use of RFID as a truly ubiquitous technology. The goal of this paper is to consider and determine the suitability of RFID for ubiquitous and ubiquitous computing by reviewing existing research in this area.

The objective of this research is to develop a comprehensive understanding of the state of the art in using RFID as a basis for the development of a generic ubiquitous framework that is suitable for rapid deployment. Through the research it is intended to consider aspects of usability of diverse smart objects by developing a demonstrator and performing validation tests with users.

\section{SMART SPACE DEVICES}

A smart device is an everyday physical object that has been enhanced by the addition of technology to create a smart device. An example of such a smart device is the smart coffee cup, MediaCup [7], which serves as a coffee cup and also pocesses additional processing, sensing and networking capability that can infer information about the state of the user.

The identification of smart objects is considered a prerequisite for developing smart behaviour, which also requires support mechanisms for; event propagation, location management, and provision of the basic services of the object. The attachment of tags to physical objects allows for automatic identification and collection of location information when brought into the proximity of a tag detection system. RFID technology has been identified as a suitable technology for such object tagging [8] to create a smart object.

RFID enhanced objects can use passive tag systems, which have a unique ID and small amounts of up to a 100 bytes of read/write memory, or active RFID systems, which have an inbuilt battery permitting transmission up to $100 \mathrm{~m}$ and a life of up to 10 years [9].

\subsection{Smart Space Characteristics}

Smart objects operating in smart environments or spaces can respond to users preferences or profiles and provide a variety of services to users. These smart 
spaces require system software and associated communications infrastructures to interact with smart objects and users. Kindberg and Fox [10] identify two key characteristics of ubiquitous systems; Physical Integration and Spontaneous Interoperation. Physical Integration is the interaction between computing nodes and the physical world. Smart objects provide the interface between the physical and the virtual world. There is a stated recognition between different environments, such as private as in the home, or public as in work spaces, and boundaries are required to demarcate these different spaces.

Spontaneous Interoperation involves components of the Smart Space that can change identity and functionality as required. This involves service discovery in which components can locate a service instance that meets their needs, bootstrapping of devices in which addresses are allocated to permit network integration of the smart devices, and interaction in which the components of the Smart Space have a common interoperation model to permit operation across environment boundaries. There are significant challenges in the development of systems that can provide the functionality of smart spaces. There is a need for appropriate frameworks to enable the development of ubiquitous environments and many frameworks based on alternative technologies have been developed. A review of frameworks that support RFID infrastructures is presented in the next sections.

\section{RFID FRAMEWORKS FOR UBIQUITOUS COMPUTING}

The development of a suitable framework requires consideration of many design concepts such as those identified in [8], these are considered suitable for discussion of the frameworks that follow in the review section.

Location, whether geographic or coordinate based, is essential in identifying the context of smart objects. This information can be readily acquired from RFID readers interacting with tagged objects. The concept of neighbourhood is related to location and is important in supporting cooperating devices in a smart environment. It is necessary to consider location management in terms of the physical object location and a symbolic location as in the case of a room in a building, both of these two aspects need to be integrated in a meaningful manner. Time is also an important aspect of any smart system in order to timestamp and order events identified by different interfaces such as an RFID reader. Related to time is the concept of history to support logging of events to support querying of past events.

In many scenarios an object may consist of many objects, such as a truck transporting smart objects, this is the concept of composition that can be an essential element of a Smart Space framework. There is a need for a link between the physical world and the virtual world, of the supporting computer system, to identify events such as tags entering or leaving the Smart Space.

A major design concern is the context of the smart device, which may depend on the presence of other devices, as applications or services are only provided 
in certain predefined circumstances. Applications or services require to assign state and behaviour to physical objects in a flexible manner to support different scenarios.

A virtual counterpart for a physical object is required to support these design concepts as the smart object has limited resources, particularly when a simple RFID tag is in use. To support the virtual counterpart, objects require to have a unique name tag and an address to permit storage in a computer environment. These virtual counterparts require to be managed from creation until their end of use and possibly beyond end of use to provide a traceable history.

An underlying requirement for applications or services in a smart environment is a communications infrastructure to permit access by smart object to the services. To support mobility in the environment a wireless based solution such as Bluetooth, WLAN or Zigbee [11].

\section{REVIEW OF ACADEMIC FRAMEWORKS}

Significant research has been carried out in to the development of frameworks to support smart spaces and smart objects. A number of these that have been developed as part of academic research are presented in this section. These frameworks are considered as academic as the papers reviewed indicate that the systems had not been deployed in the real world for long periods to permit validation by users.

\subsection{Intuitive Service Discovery in RFID Enhanced Networks}

In [12] Antoniou et al. present an Intuitive Service Discovery architecture that supports the interaction between smart devices, such as an IP network printer enhanced with an RFID tag. The RFID technology permits service discovery to occur through touch gestures by the user devices, which are mobile RFID devices using wireless connections. RFID was chosen, as it is well suited to automatic service discovery and configuration. To encourage this approach of RFID enhanced contact-less short-range interaction the Near Field Communications (NFC) Forum [13] was established.

The proposed framework permits the automatic discovery of a printer by a new user in a shared Smart Space. An RFID enhanced mobile phone is gestured at a printer and this connects the phone to the local WLAN to permit use of the printer by the phone. This framework was designed to operate with a number of service discovery technologies such as UPnP [14] or Web Services.

This framework requires a significant level of infrastructure in the form of WLAN, DNS and DHCP servers. In many public hotspots this infrastructure is readily available and would provide mobile users with the capability to select a suitable smart device with which to interact.

\subsection{Service Framework with RFID and Sensor Networks}

An alternative approach to developing a Smart Space is through the use of Wireless Sensor Networks (WSNs), which are small devices with processing ca- 
pability, sensor inputs and an RF transceiver for setting up a communications infrastructure, such as in [15] for monitoring the temperature in refrigerated lorries. In [16] Lopez et al identify a novel approach of integrating RFID technology with a Wireless Sensor Network (WISSE). They propose a cluster based approach for organizing the entities, smart objects and identify how the communication between groups is maintained using a particular sensor node that acts as a connection, referred to as a correspondent, between clusters. A prototype implementation of the framework is presented that uses a PDA and RFID device and operates over WiFi rather than a true WSN technology as shown in Figure 1.

This framework uses a centralized approach to maintaining information about physical devices and virtual devices, in order to minimize communication overhead in the network. This has limitations in terms of scalability, particularly as WSNs are generally used in a environment with many hundreds of sensor nodes in the network.

\subsection{Active and Passive Tags for Spontaneous Interaction}

Siegemund and Florkemeier [17] present an interesting set of 3 scenarios using smart objects based on Bluetooth enabled active RFID tags, BTnode [18]. The interaction between the different smart objects and users is enabled through the use of mobile phones using Bluetooth when users and objects are close and SMS when the distance is too long for Bluetooth. A conceptual overview of the system is shown in Figure 2.

In each scenario in [17] the active tags are used to enhance normal objects. In the case of the egg box the tag monitors the condition of the eggs in the box to determine whether the eggs are intact or broken. In their smart medicine cabinet design, the cabinet reminds users when it is time to take their medicine, by sending an SMS message. It keeps track of the contents of the cabinet by using the an RFID reader, within the cabinet, that reads the serial number of the RFID tags on medication boxes. In the remote interaction case a user can use their phone to call an object and query the state of the object such as to determine who is in a particular smart office. In this approach they define an architecture to support the operation of the different applications and identify the potential for using passive and active RFID tags together. In particular they identify the ability of attaching an RFID scanner to an active tag to permit active and passive RFID tags to be integrated.

\subsection{The Smart Container}

Floerkemeier et al. [19] address the issues of monitoring the contents of everyday containers using RFID. The inconvenience of monitoring the contents of containers combined with the possibility of human error creates the need for such a solution. This demonstration is called the smart box. The smart box is an example of self-contained RFID application, which does not communicate with external sensors. 


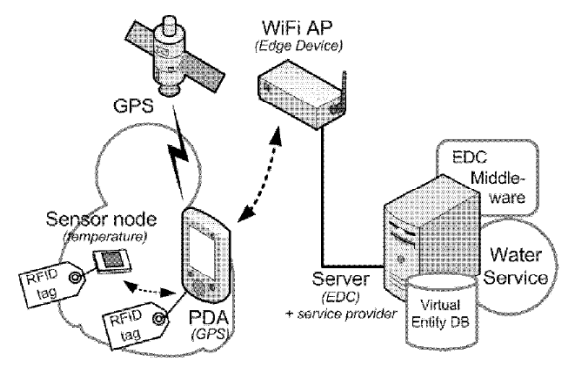

Fig. 1. WISSE Implementation Scenario

Examples of real world boxes which must be monitored are the toolbox, the first aid kit, and the medicine cabinet. Since these boxes may be checked many times with no abnormality being identified, this repetitive task can become irritating. Since the purpose of this application is to remove inconvenience or annoyance, the application is designed in such a way that it avoids further irritation by permitting people to interact with the container in their usual manner. The proposed smart box offers the following functionality:

- The contents of the box must be monitored unobtrusively

- The desired configuration of the box must be known and any discrepancy must be indicated.

With the above functionality implemented, the implementation of additional services would be possible such as provision of detailed information on items and a mechanism for identifying which person is currently interacting with the container.

Two possible interfaces are proposed for the system. The most simple interface is a indicator light, which turns red or green depending on the status of the box. A more detailed interface is also proposed which displays information about the box on a LCD screen. This information includes a list of the items are in the box, which items are missing, if any, and a usage history of each item. When a user adds or removes an item from the box, additional information about this item will be displayed on the LCD screen. Three sample smart box applications were discussed in [19], a smart toolbox, a smart medicine cabinet and a smart surgical kit.

Smart Toolbox The smart toolbox was implemented to support aircraft maintenance mechanics in their daily jobs. The system enables automatic monitoring of toolboxes. This prevents situations such as missing tools or a situation in which tools are returned to a toolbox which they do not belong to. A warning is also displayed if the person interacting with the box is not the owner of that box. An RFID tag is attached to each tool and the box itself is equipped with and RFID reader and antenna. 


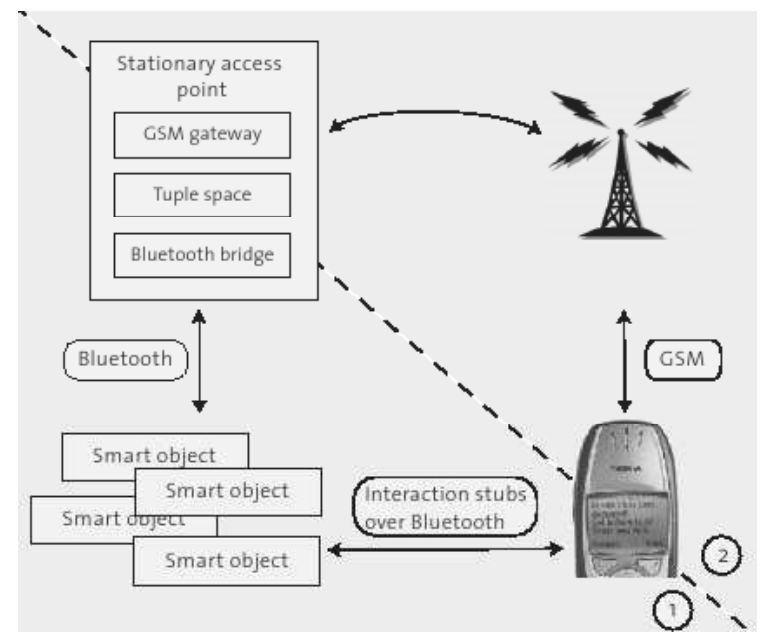

Fig. 2. Overview of the main architectural components in the remote interaction and smart product monitoring scenario: when the user is in range of a smart object, interaction stubs are transferred to his/her mobile phone (1), when far away, communication takes place over the cellular phone network (2).

Smart Medicine Cabinet This application is used to track the contents of the medicine cabinet and to ensure that necessary prescription medication is contained within the cabinet at all times. The system is capable of tracking expiry dates on drugs and hence prevents situations where patients accidentally continue to use medication beyond its expiry date. Accessibility features are also present in the system such the ability to view and manage the contents of the cabinet remotely via a mobile phone or for the visually impaired who may have difficulty reading small print on prescription bottle, the system can display the name of the item on the LCD screen when it removed from the cabinet and a synthesized voice informs the user of the name of the medication.

Smart Surgical Kit The third demonstration of the smart container was in the form of a smart surgical kit. The key problem which this application addresses is the case in which a surgeon forgets to remove swabs and bandages used during surgery. Currently, this problem is addressed by post-surgery X-Rays. This not only creates the inconvenience of needing X-Rays, but also the issue of opening a wound to retrieve a misplaced swab. For this application, it is necessary to monitor both the contents of the surgical kit and the bin into the which the used swabs are placed. Each swab is tagged with an RFID chip to monitor its location. Any items not in either the waste bin or the surgical kit can be assumed to be in use.

Floerkemeier et al. [19] felt that the smart surgical kit and the smart medicine cabinet exhibit particular promise. The smart toolbox, while an interesting ap- 
plication was perhaps the least useful of the three. It could be used as interesting showcase for RFID technology but its use in everyday life is questionable, at least until the cost of such technology is further reduced.

\subsection{Smart Playing Cards}

The smart playing cards application [20] applies RFID technology to an existing card game, Whist. This approach is presented as differing from many applications of technology to gaming where new games are developed to exploit or to showcase the capabilities of technology.

The game works by using standard playing cards to which adhesive RFID tags have been attached. The cards are laid on a table, as normal, but in this instance the table has an RFID antenna mounted such that cards are detected as they are played.

The system developed can determine the winning card in each trick played, can detect if a player's move would be illegal, and notify accordingly, and can also keep score.

Further concepts presented include the development of a training system for beginners to indicate whether a card played was a good or bad choice, and ultimately to prompt the player based on previous hands played. The rules of Whist are very straightforward, but a suitable extension of this project would be to develop systems to support other card games, and to assist the novice in learning the game.

The fluidity of the playing of the real game was compromised by the reaction speeds of the system. The speed at which the antenna could detect the removal of cards from the table, for example, caused a latency which experienced players would not tolerate.

Another limitation had to do with the RF properties of the antenna. The antenna has a sensitivity pattern of a hemisphere of diameter equal to the length of the antenna. In certain circumstances, this sensitivity is such that it can cause false detections of cards that have not yet been played. The proposed solution is to use a series of smaller antennae resulting in a 'lower' sensitivity bubble.

\subsection{RFIDChef}

In [21] Langheinrich et.al. refer to the RFID Chef project. This project is based in a household kitchen. The co-location of tagged ingredients on a smart surface allows the system to retrieve recipes appropriate to the ingredients available. This system was developed in order to explore issues including; the application of RFID technologies to non-technical environments, a greater range and number of interacting artefacts when compared with other implemented RFID applications and an increase in the interaction complexity. A schematic view of the RFID Chef hardware is shown in Figure 3. 
The software system comprises of a a number of layered software modules implemented in a range of languages (C, Java and Python).

- Sensory Control and Input Processing Module to poll sensors.

- Basic Event Modelling Module which aggregates lower level events provided by the above module to generate events with a larger granularity.

- Context Event Modelling Module to improve the signal/noise ratio by applying a time-based filter to the detected events.

These modules serve to reduce jitter in the system due to the apparent arrival and departure of tags from the system. This jitter is most likely due to the nature of the RFID technology where tags may appear to arrive and depart the system while in reality the have not moved. This jitter also manifests itself when objects genuinely do move in and out of the range of the sensor antenna due to the chef's rearranging of utensils, or ingredients in the kitchen.

\subsection{Smart Libraries}

The use of RFID in libraries has been widely written about $[22,23]$. An interesting use of RFID in libraries was the provision of digital library services [24] to mobile users. This system aims to eliminate the use of large desktop computers as a searching facility in libraries and to replace them with mobile devices such as PDAs and smart phones. This allows users to identify and view information on both physical and non physical library items on their mobile device. This can be achieved, it is proposed, through the use of active RFID tags as a means of storing meta data about each object in the library in conjunction with the use of RFID enabled mobile devices.

Each library item contains an active RFID tag which contains information about the object in question. This data can then be transmitted to a range of devices. This system uses two types of RFID-based query services: on-demand or broadcast. If query information is broadcast from the RFID tag, it is the responsibility of the mobile host to recognize the information as relevant and retrieve it. On-demand queries can be real time or delayed. Real-time RFID queries will retrieve information immediately while delayed queries will allow the mobile host to voluntarily disconnect, process the query, and transmit the result when the mobile host reconnects. For efficiency purposes an RFID caching proxy is also used to store the results of frequent queries. The system is also capable of scaling down results based on bandwidth availability and the specification of the mobile device in question.

The framework for the smart library is an interesting approach and could be used to deliver ubiquitous digital library services across a broad range of devices. Security issues when using RFID in libraries as described by Molnar [25] would also need to be considered in such a framework.

\section{REVIEW OF DEPLOYED FRAMEWORKS}

This section provides a review of RFID based frameworks that have been deployed and are in use in applications in museums and educational settings. These 
examples permit the usability of the technologies to be assessed through feedback from users.

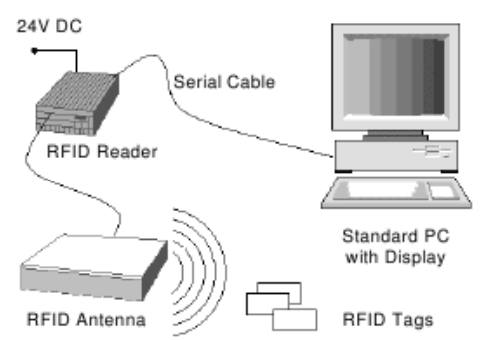

Fig. 3. Schematic view of the RFID Chef hardware. The RFID reader antenna detects tagged artifacts in its vicinity and transmits raw sensory information through a reader module to the PC via serial cable.

\subsection{RFID in Museums}

eXspot $[26,27]$ is a custom-designed RFID application being prototyped and evaluated over the past three years at the Exploratorium museum in San Francisco. Using RFID tagged visitor cards, visitors can trigger automatic cameras to take photographs and capture information about exhibits on display at the museum. This allows bookmarking of exhibits, which is a feature which visitors have previously expressed an interest in [28]. The system eliminates the need for the user to carry additional items such as cameras and informational booklets. Visitors can later use their RF tagged cards to log onto kiosks which allow them to view photographs which they have taken. They can continue their exploration either from museum kiosks or from home by logging onto a personalized web page which shows the dates they were at the museum, which exhibits they visited, photographs taken, as well as links to additional educational information on exhibits which they bookmarked.

The system consists of small RFID readers attached to museum exhibits, RFID tagged cards carried by visitors, a wireless network, a registration kiosk and dynamically generated webpages. An RFID reader unit is located at each exhibit with a control processor and radio connectivity, a low-power RFID reader with a range of a few inches, and LEDs which show the systems state to visitors. Visitors hold their card in the vicinity of the eXpsot RFID reader, which interrogates their card to read an ID number which is then sent wirelessly to a base station. The base station then records the ID number, time of visit and exhibit information for access by the visitor at a later occasion.

The Exploratorium [26] is not the only museum to adopt the use of RFID. The museum of Science and Industry in Chicago have opened an exhibition 
called "NetWorld" where visitors learn about the Internet. Users design personal avatars before being given an RFID tagged visitor card. The personalized avatars then accompany and interact with them throughout their visit as they learn about the Internet and how it works. The Museum of Natural History in Aarhus, Demark takes a slightly different approach for their exhibit named "Flying". Stuffed birds are tagged with RFID chips and visitors carry RFID readers which they can use to scan the birds allowing them to view a presentation of related text, quizzes, audio and video [27].

The use of RFID in educational environments can provide a rich learning experience and promote user curiosity. RFID has also been used in more traditional learning environments such as the lecturer theater. Transnote is one such example.

\subsection{Transnote}

Lecturers in the Japan Advanced Institute of Science and Technology are using RFID to display students' notes on a shared media board via the Transnote [29] system. The concept of shared media boards has been widely considered as in [30], but in this example RFID technology is employed as the key enabling technology. The system provides a note sharing facility, which allows users to share notes, written on regular paper, using a shared media board. This is enabled through the use of digital pens and wireless communications. Initially, a lecturer used a desktop computer to control the contents of the shared media board. However, it was noted in a user survey that this proved to be an inconvenience since it is more natural for the lecturer to walk around the class and interact with the students. In addition to this, not all lecturers were comfortable with the use of a PC, which created the need for additional training on the lecturer's part to familiarize themselves with the interface of the new system. To solve this problem an RFID remote was introduced.

The remote takes the form of a PDA with an RFID reader attached. As the lecturer walks around the class they may see a set of notes they wish to show to the entire class. To achieve this, the lecturer simply holds the remote over the students RFID tagged digital note taking facility. Once the student tag is identified by the lecturer's RFID reader, a command can be sent to the manager to display the corresponding notes. At this point, the lecturer may ask the student to explain the notes to the class. The student will typically explain the notes by pointing and drawing with their pen. To support this, the system uses a tracking function which supports zooming and positioning of the notes to correspond with the movement of the students pen.

The use of RFID in this system allows for a more natural approach. It permits a lecturer to work in a fashion they are accustomed to and comfortable with, and allows students to see the lecturer approach and prepare them for having their notes displayed. One point noted is that the apparatus used by the student for note taking leaves something to be desired in terms of size. 


\section{ANALYSIS AND REVIEW}

\subsection{Physical Integration}

The use of the passive RFID tags permits significant physical integration of the smart devices. In particular the approach of using both passive and active tags [17] to monitor the items in a cabinet is quite innovative. It permits the use of inexpensive tags on many individual items and requires only one more costly active tag to be used to create a range of adaptable solutions. In particular the use of SMS as a mechanism for sending event alerts is practical and can be readily implemented with existing telecom infrastructure.

\subsection{Spontaneous Interoperation}

Each of the Frameworks present a differing approach to solving the issues of spontaneous operation and in particular to providing service discovery for users in the Smart Space.

The simplest approach identified in [8] use passive tags in each case and provide a usable approach that is inexpensive. However in the case of smart tool box the prototype application has been developed to operate as a standalone unit. A communications infrastructure would be useful to permit the collection of data and identification of event alerts that could be circulated.

\subsection{Academic Versus Deployed Frameworks}

The academic frameworks provide indications of the potential uses of RFID and suggest approaches for addressing the issues of Physical Integration and Spontaneous Interoperation. The implementations identified as academic have not been validated by significant user interaction and usability testing. In order to determine the true potential of RFID technologies these frameworks require more exhaustive testing. The prototype systems used in such academic research, as in $[19,8]$, may not readily translate into a real world implementations as the level of physical integration could be limited and thus would not be considered as truly ubiquitous solutions.

The deployed frameworks such as the museum example [26] have been used in real world situations and have been evaluated by users. Spontaneous interoperation is supported in these deployed cases as the systems can be observed in operation and do provide services autonomously. The user devices, RFID cards, also indicate the concept of physical integration through the size and operation. However they identify the need for diverse collaborating technologies to create a suitable infrastructure.

The deployed frameworks provide the ability to gain user feedback and permit modification of frameworks and the system configuration to meet usability requirements. This was particularly clear in the Transnote [29] case where the system was modified to match users' normal lecturing styles. Through deployment the issue of user training also became apparent, which is often overlooked during an academic research activity. 


\section{CONCLUSION}

This review underlines the case for RFID as a ubiquitous technology to support the development of smart spaces. A number of indicative examples of the use of RFID technologies for developing ubiquitous environments were presented. The review of the academic frameworks gives an indication of the potential applications of RFID in the development and provision of smart environments and spaces. However these approaches have limitations in that they have yet to be validated in large scale user tests. Also, before RFID can become a truly ubiquitous technology, there are still many research challenges to be faced. Such challenges include security, privacy, deployment challenges such as health and safety and aesthetics, as well as technical challenges such as system failures and input data errors. For example, duplicate tag readings have been discussed by Welbourne et al. [31], which involve the same tag being detected by multiple antennas simultaneously or several times by the same antenna in a very short time interval. Cleaning RFID data is a active area of research $[32,33]$ which addresses such problems. The deployed frameworks address the real challenge, user acceptance, in creating viable smart spaces and usable smart objects.

To meet Weisers' concept of disappearing computing and technologies [5] requires that users are willing to accept the use of that technology into their everyday lives. This level of acceptance requires non-invasive technologies which are intuitive, self organizing, self managing and which require minimal interaction by the user.

\section{FUTURE WORK}

The next stage in our research program is the development of a demonstrator system using RFID technologies and the development of an overarching framework that will address the limitations of existing solutions. The demonstrator will involve the deployment of a Smart Space across an academic campus to support lectures and instant tutorials for local and remote access by participants to both pre-scheduled and ad-hoc lecturing events. In particular the research will focus on the use of Near Field Communications (NFC) solutions, with a core aim to minimize complexity in order to achieve an optimal design for rapid deployment and intuitive user operation.

\section{Acknowledgements}

Dan MacCormac gratefully acknowledges the contribution of the Irish Research Council for Science, Engineering and Technology: funded by the National Development Plan.

\section{References}

1. Wamba, S.F., Lefebvre, L.A., Lefebvre., E.: Enabling intelligent B-to-B eCommerce supply chain management using RFID and the EPC network: A case study in 
the retail industry. In: The 8th international conference on Electronic commerce. (2006) 281-288

2. Srivastava, B.: Radio Frequency ID technology: The next revolution in SCM. Business Horizons 47/6 (2004) 60-68

3. EPC Global (2006) [Online] Available: http://www.epcglobalinc.org

4. Roberti, M.: Analysis: RFID - Wal-Mart's network effect. CIO Insight (2006)

5. Weiser, M.: The computer for the 21st century. Scientific American 265/ 3 (1991) 94-104

6. Mangione-Smith, W.H.: Mobile computing and smart spaces. IEEE Concurrency 6 / 4 (1998)

7. Beigl, M., Gellersen, H., Schmidt, A.: Mediacups: Experience with design and use of computer-augmented everyday objects. Computer Networks 35/4 (2001) 401-409

8. Römer, K., Schoch, T., Mattern, F., Dübendorfer, T.: Smart identification frameworks for ubiquitous computing applications. Wireless Networks 10 (2004) 689-700

9. Philips ICODE smart label solutions. [Online] Available: http://www.philips.semiconductors.com/products/identification/icode/index.html

10. Kindberg, T., Fox, A.: System software for ubiquitous computing. IEEE Pervasive Computing 1 / 1 (2002) 70-81

11. Baker, N.: Zigbee and bluetooth strengths and weaknesses for industrial applications. Computing \& Control Engineering Journal 16 / 2 (2005) 20-25

12. Antoniou, Z., Krishnamurthi, G., Reynolds., F.: Intuitive service discovery in RFID-enhanced networks. In: Proceedings of IEEE COMSWARE Conference. (January 2006)

13. NFC forum [Online] Available: http://www.nfc-forum.org

14. UPnP forum [Online] Available: http://www.upnp.org

15. Shan, Q., Liu, Y., Prossec, G., Brown., D.: Wireless intelligent sensor networksfor refrigerated vehicle. In: IEEE 6th CAS Symposium on Emerging Technologies: Mobile and Wireless Communication. (2004)

16. Lopez, T.S., Kim, D., Park, T.: A service framework for mobile ubiquitous sensor networks and RFID. 1st International Symposium on Wireless Pervasive Computing (2006) 16-18

17. Siegemund, F., Florkemeier, C.: Interaction in pervasive computing settings using bluetooth-enabled active tags and passive RFID technology together with mobile phones. In: The First IEEE International Conference on Pervasive Computing and Communications (PerCom). (2003)

18. Beutel, J., Kasten., O.: A minimal bluetooth-based computing and communication platform. Technical report, Computer Engineering and Networks Lab, Swiss Federal Institute of Technology (ETH) Zurich (2001)

19. Floerkemeier, C., Lampe, M., Schoch, T.: (The smart box concept for ubiquitous computing environments)

20. Romer, K., Domnitcheva, S.: Smart playing cards: A ubiquitous computing game. Personal and Ubiquitous Computing 6 (2004) 371-377

21. Langheinrich, M., Mattern, F., Rmer, K., Vogt, H.: First steps towards an eventbased infrastructure for smart things. Technical report, ETH Zurich, Swiss Federal Institute of Technology (2000)

22. Boss, R.W.: RFID technology for libraries (2004)

23. Smart, L.: Making sense of RFID. Netconnect (Fall, 2004) (2004) 4-14

24. Morales-Salcedo, R., Ogata, H., Yano, Y.: Towards a new digital library infrastructure with RFID for mobile e-learning. In: IEEE International Workshop on Wireless and Mobile Technologies in Education (WMTE05). (2005) 
25. Molnar, D., Wagner, D.: Privacy and security in library RFID issues, practices, and architectures. In: Conference on Computer and Communication Security. (2004)

26. Hsi, S., Semper, R., Brunette, W., Rhea, A., Boriello, G.: eXspot: A wireless RFID transceiver for recording and extending museum visits. In: Proceedings of UbiComp. (2004)

27. Hsi, S., Fait, H.: RFID enhances visitors' museum experience at the Exploratorium. Communications of the ACM 48 / 9 (2005) 60-65

28. Fleck, M., Frid, M., Kindberg, T., Rajani, R., OBrien, E.: From informing to remembering: Deploying a ubiquitous system in an interactive science museum. IEEE Pervasive Computing 1 (2002) $13-21$

29. Miur, M., Kunifuji, S., Shizuki, B., Tanaka, J.: Airtransnote augmented classrooms with digital pen devices and RFID tags. In: IEEE International Workshop on Wireless and Mobile Technologies in Education (WMTE05). (2005)

30. Elrod, S., Bruce, R., Gold, R., Goldberg, D.: Liveboard: A large interactive display supporting group meetings, presentations, and remote collaboration. In: Conference on Human Factors in Computing Systems. (1992)

31. Welbourne, E., Balazinska, M., Borriello, G., Brunette, W.: Challenges for pervasive rfid-based infrastructures. In: PERCOMW '07: Proceedings of the Fifth IEEE International Conference on Pervasive Computing and Communications Workshops, Washington, DC, USA, IEEE Computer Society (2007) 388-394

32. Jeffery, S.R., Garofalakis, M., Franklin, M.J.: Adaptive cleaning for rfid data streams. In: VLDB '06: Proceedings of the 32nd international conference on Very large data bases, VLDB Endowment (2006) 163-174

33. Khoussainova, N., Balazinska, M., Suciu, D.: Towards correcting input data errors probabilistically using integrity constraints. In: MobiDE '06: Proceedings of the 5th ACM international workshop on Data engineering for wireless and mobile access, New York, NY, USA, ACM (2006) 43-50 